\title{
Freqüência de alterações cérvico-vaginais em mulheres submetidas ao exame citopatológico
}

\author{
Frequency of cervico vaginalis alteration in submitted patients of cytopathological exam \\ Frecuencia de las alteraciones cervicovaginal en mujeres sometidas al examen \\ citopatógico
}

\begin{abstract}
Janaína Valadares Guimarães', Ana Karina Marques Salge", Flávia Aparecida de Oliveira'"I, Ruy de Souza Lino Júnior ${ }^{\vee v}$, Eumenia Costa da Cunha Castro ${ }^{\vee}$, Marlene Antônia dos Reis ${ }^{\mathrm{V}}$, Vicente de Paula Antunes TeixeiravII
\end{abstract}

\begin{abstract}
RESUMO
O Exame citopatológico é o método de triagem que auxilia na detecção precoce do câncer e de lesões pré-cancerosas da cérvix. No Brasil, o câncer cervical representa a segunda neoplasia maligna mais incidente em mulheres. $O$ objetivo desse estudo foi avaliar a freqüência das alterações citopatológicas de acordo com a faixa etária. A seleção dos casos foi realizada através dos resultados dos exames citopatológicos, de mulheres na faixa etária entre 14 a 80 anos, obtidos no Centro de Atenção Integrada à Saúde da Mulher, Uberaba (MG), no período de 2001 a 2003. Dos 35.220 resultados obtidos, em 3,34\% foram descritos alterações no epitélio cérvico-vaginal, sendo mais freqüentes as alterações escamosas e glandulares de significado indeterminado $(53,7 \%)$ e as infecções pelo HPV $(31 \%)$. As mulheres mais jovens (< 40 anos) foram significativamente mais acometidas por essas alterações $(p<0,005)$. Neoplasias invasivas acometeram $5,6 \%$ das mulheres a partir da quarta década de vida $(p<0,005)$. Os diagnósticos das alterações no epitélio cérvicovaginal de acordo com a faixa etária das mulheres foram semelhantes aos descritos na literatura, sendo que infecção pelo HPV, principal agente potencialmente carcinogênico, acometeu mulheres mais jovens $e$ as neoplasias invasivas foram mais freqüentes a partir da quarta década de vida.
\end{abstract}

Palavras chave: Diagnóstico; Exame citopatológico; Saúde da mulher.

\section{ABSTRACT}

The cytopathological exam is useful recommended for early diagnoses of neoplastic and of pre-neoplastic lesions of the uterine cervix. In Brazil, cervix neoplasm is the second most frequent malignant neoplasm in women. The aim of this study was evaluate the frequency of cytopathologic alterations and its relationship with age. The cases selection was performed by means of cytopathological exam results in women aged 14 to 80 years old, by the revision in Centro de Atenção Integrado a Saúde da Mulher, Uberaba (MG), during the period of 2001-2003. From de 35.220 reviewed exams, $3.34 \%$ tests showed alterations in the cervico vaginalis epithelium, with a higher frequency in the squamous cervical epithelium alterations and glandular epithelium alterations of unknown significance $(53.7 \%)$ as well as infection by the Human Papillomavirus (HPV) $(31 \%)$. The infections caused by the HPV and atypical glandular and scamous cells of undetermined significance were more frequent in ages under the $40 \quad(p<0.005)$, yet the occurrence of cervical carcinoma was more frequent in women over $40 \quad(p<0.005)$. Therefore, the frequency of cytopathologic alterations of the cervical and vaginal epithelium and its relationship to age were

\footnotetext{
Enfermeira. Doutora em Ciências da Saúde. Professor Adjunto da Disciplina de Patologia Geral do Instituto de Patologia Tropical e Saúde Pública da Universidade de Goiás. janaina@iptsp.ufg.br

"Enfermeira. Doutora em Ciências da Saúde. Professor Adjunto da Faculdade de Enfermagem da Universidade Federal de Goiás. anakarina@fen.ufg.br

III Enfermeira. Doutora em Ciências da Saúde. Professor Adjunto da Disciplina de Patologia Geral do Instituto de Patologia Tropical e Saúde Pública da Universidade Federal de Goiás. flavia@iptsp.ufg.br

IV Enfermeiro. Doutor em Ciências da Saúde. Professor Adjunto da Disciplina de Patologia Geral do Instituto de Patologia Tropical e Saúde Pública da Universidade Federal de Goiás. ruy@iptsp.ufg.br

$\checkmark$ Médica. Doutora em Patologia. Professor Adjunto da Disciplina de Patologia Geral da Universidade Federal do Triângulo Mineiro. eumenia.pat@dcb.uftm.edu.br

vi Médica. Doutora em Patologia. Professor Adjunto da Disciplina de Patologia Geral da Universidade Federal do Triângulo Mineiro. mareis.pat@dcb.uftm.edu.br

VII Médico. Doutor em Patologia. Professor Titular da Disciplina de Patologia Geral da Universidade Federal do
} Triângulo Mineiro. vicpat@dcb.uftm.edu.br 
resemblance to described in literature. The HPV infection, the best carcinogenic potential agent attacked younger patients and the invasive neoplasm was more frequent in women over 40 years old.

Key words: Diagnostic; Cytopathological exam; Woman health.

\section{RESUMEN}

El exámen citopatológico es un método de triage que ayuda a realizar una detección precoz del cáncer y de lesiones pre-cancerosas del cérvix uterino. En el Brasil, el cáncer cervical representa la segunda neoplasia maligna mas incidente en mujeres. El objetivo del estudio fue avaliar la frecuencia de las alteraciones citopatológicas de acuerdo con el grupo etario. La selección de los casos fue realizada a través de los resultados de los exámenes citopatológicos, de mulheres dentro del grupo etario entre 14 a 80 años, obtenidos del Centro de Atención Integrada a la Salúd de la Mujer, Uberaba (MG), durante el período del

\section{NTRODUÇÃO}

O carcinoma cervical (CC) compreende $12 \%$ de todas as neoplasias malignas, sendo o segundo mais comum em mulheres. Em 2000, 417.000 novos casos foram diagnosticados e ocorreram 55,97\% de óbitos em todo mundo. Cerca de $80 \%$ desses óbitos ocorreram em mulheres de paises em desenvolvimento ${ }^{(1)}$.

Nos últimos 50 anos, modificações clínicas e epidemiológicas contribuíram para a diminuição da morbidade e mortalidade por CC na China ${ }^{(2)}$. A avaliação realizada antes e após o programa de prevenção, em mulheres com idade entre 24 a 64 anos, demonstrou diminuição dos casos de óbitos por CC de $12.8 \%$ em 1980 para $6.8 \%$ em $2001^{\text {(3). }}$

No México, um estudo transversal direcionado a mulheres em idade fértil, enfatiza a necessidade de ampliação da promoção à saúde para mulheres de alto risco, incluindo os seus parceiros sexuais ${ }^{(4)}$.
2001 al 2003. De los 35.220 resultados obtenidos, en $3,34 \%$ fueron descritas alteraciones en el epitélio cervico-vaginal, siendo mas frecuentes las alteraciones escamosas e glandulares de significado indeterminado $(53,7 \%)$ y las infecciones por el HPV (31\%). Las mulheres más jóvenes $(<40$ años) fueron significativamente más acometidas por esas alteraciones $(p<0,005)$. Así, las neoplasias invasivas acometieron al $5,6 \%$ de las mulheres a partir de la cuarta década de vida $(p<0,005)$. Los diagnósticos de las alteraciones del epitélio cérvico-vaginal de acuerdo con el grupo etario de las mulheres fueron semejantes a otros hallazgos ya descritos en la literatura, siendo que la infección por el VPH, principal agente con potencial carcinogénico, acometió a mulheres más jóvenes y las neoplasias invasivas fueron más frecuentes a partir de la cuarta década de vida.

Palabras clave: Diagnóstico; Examen citopatológico; Salúd de la mujer.

Na África do Sul, mulheres submetidas ao exame citopatológico apresentaram uma diminuição em torno de $70 \%$ do risco de desenvolverem CC, demonstrando a importância dos programas de prevenção, especialmente nas regiões onde essa técnica foi pouco difundida ${ }^{(5)}$.

Os fatores socioeconômicos relacionados ao CC foram analisados em mulheres com mais de 65 anos nos Estados Unidos da América. Este estudo demonstrou que a condição socioeconômica desfavorável associada à ocorrência de co-morbidades junto ao CC contribuiu de maneira significativa para uma menor sobrevida dessas mulheres ${ }^{(6)}$.

No Brasil, a estimativa de novos casos de CC em 2006 foi de 19.260, com risco de 20 casos a cada 100 mil mulheres ${ }^{(7)}$. Em 1996, o Ministério da Saúde (MS), por intermédio do Instituto Nacional de Câncer (INCA), implantou no Brasil o Programa Viva Mulher tendo como 
população-alvo, mulheres entre 35 a 49 anos. A partir de 1998, as ações de controle do CC passaram a atender mulheres com idade de 25 a 59 anos, ampliando a assistência às mulheres pelo programa ${ }^{(8)}$.

O CC guarda estreita relação com a infecção pelo Papiloma Vírus Humano (HPV). Fato amplamente difundido através de estudos que utilizaram técnicas de biologia molecular, onde os diversos subtipos do HPV foram descritos. Alguns mais persistentes e agressivos do que outros com alta capacidade evolutiva para o CC ${ }^{(9-10)}$.

Os principais fatores de riscos envolvidos na infecção por HPV incluem número de parceiros sexuais, início precoce da vida sexual, tabagismo, uso de contraceptivo oral, inflamação cervical crônica, doença imunossupressora e gravidez ${ }^{(11)}$.

O exame citopatológico apresenta alta sensibilidade, mas baixa especificidade. Devido a sua alta sensibilidade, praticamente todas as mulheres com alterações seriam detectadas, apesar de alguns resultados falsos-positivos. Por ser altamente sensível é o teste de escolha para programas de rastreamento de CC ${ }^{(12)}$.

Por outro lado, tendo em vista a importância desses dados para o planejamento da assistência à saúde da mulher, o objetivo foi demonstrar a freqüência das alterações citopatológicas no exame citopatológico comparando as faixas etárias das mulheres nos anos de 2001 a 2003, em Uberaba, MG.

\section{MÉTODOS}

Estudo retrospectivo, realizado em 2006, em 35220 resultados de exames citopatológicos, utilizando dados referentes ao período de janeiro de 2001 a agosto de 2003, das Unidades Básicas de Saúde (UBS) e do Centro de Atenção a Saúde da Mulher (CAISM) da Secretaria Municipal de Saúde (SMS), em Uberaba, MG. O projeto foi submetido e aprovado pelo Comité de Ética em Pesquisa da UFG (protocolo $\mathrm{n}^{\circ}$ 034/2006).

A coleta desses exames foi realizada por profissionais de saúde das UBS e do CAISM, na sua maioria enfermeiros. Para a realização desse estudo todos os resultados dos exames citopatológicos cérvico-vaginais satisfatórios foram incluídos neste estudo. As informações referentes à idade e ao diagnóstico citopatológico foram obtidas através da revisão dos resultados dos exames arquivados no banco de dados do CAISM. Os diagnósticos citopatológicos descritos foram: infecção pelo HPV, alteração em células escamosas e glandulares de significado indeterminado (ASCUS-AGUS), neoplasias intraepiteliais cervicais de baixo grau, moderada, acentuada ( NIC I, II e III) respectivamente e CC.

As mulheres apresentaram idade variando de 14 a 80 anos, e foram divididas em dois grupos, com menos de 40 anos $(<40$ anos) e com mais de 40 anos ( $\geq 40$ anos). Após a obtenção dos resultados dos exames citopatológicos, comparamos a freqüência dos diagnósticos em relação às faixas etárias dos grupos de mulheres avaliadas. Para isso, uma planilha eletrônica foi elaborada para o processamento e a análise dos dados. A análise estatística foi realizada com o programa "SigmaStat 2.03". As variáveis foram testadas para verificar se houve distribuição normal através do teste de Kolmogorov-Sminov. As proporções foram comparadas pelo teste do chi-quadrado $\left(\chi^{2}\right)$. As diferenças foram 
consideradas estatisticamente significantes quando $p<0,05$.

\section{RESULTADOS}

As alterações descritas no epitélio cérvico-vaginal (ECV) representaram 3,34\% (1176) do grupo total, sendo que as mulheres com menos de 40 anos apresentaram $72,4 \%$ (852) das alterações e as mulheres com mais de 40 anos 27,6\% (324).

As ASCUS-AGUS e a infecção pelo HPV foram as alterações mais descritas, com uma freqüência de 46,4\% (546) e 31,0\% (365) dos casos, respectivamente. Uma diferença estatisticamente significativa foi encontrada entre os grupos de mulheres com menos de 40 anos e 40 anos ou mais com diagnóstico de ASCUS-AGUS $\left(\chi^{2}=3,891, p=0,049\right)$ e infecção por HPV $\left(\chi^{2}=9,687, p=0,002\right)$, onde as mulheres com idade entre 14 a 40 anos apresentaram maior freqüência dessas alterações (Tabela 1).

Tabela 1: Resultados dos Exames citopatológicos de 1176 mulheres avaliadas em 2001-2003, idade entre 14 a 80 anos, que apresentaram infecção por HPV, ASCUS-AGUS, NIC I, NIC II, e CC,

Uberaba-MG, 2006.

\begin{tabular}{cccccc}
\hline I dade (anos) & $\mathbf{1 4}$ a $\mathbf{3 9} \mathbf{n ~ ( \% )}$ & $\mathbf{4 0}$ a $\mathbf{8 0} \mathbf{~ n ~ ( \% ) ~}$ & Total $\mathbf{~ ( \% ) ~}$ & $\chi^{\mathbf{2}}$ & $\mathbf{P}$ \\
\hline ASCUS-AGUS* & $380(69,6)$ & $166(30,4)$ & $546(46,4)$ & 3,891 & 0,0049 \\
HPV** & $287(78,6)$ & $78(21,4)$ & $365(31,0)$ & 9,687 & 0,002 \\
NIC I & $116(78,9)$ & $31(21,1)$ & $147(12,5)$ & 3,155 & 0,076 \\
NIC II & $34(72,3)$ & $13(27,7)$ & $47(4,0)$ & 0,0224 & 0,881 \\
NIC III*** & $24(57,1)$ & $18(42,9)$ & $42(3,6)$ & 4,348 & 0,037 \\
CC**** & $11(37,9)$ & $18(62,1)$ & $29(2,5)$ & 16,875 & 0,001 \\
Total $(100 \%)$ & 852 & 324 & 1176 & & \\
\hline
\end{tabular}

Os diagnósticos de NIC corresponderam a $20,1 \%$ das alterações descritas, sendo $12,5 \%$ (147) NIC I, 4\% (47) NIC II e 3,6\% (42) NIC III. As mulheres com menos 40 anos apresentaram uma freqüência significativamente maior de NIC III do que as mulheres com maior faixa etária $\left(\chi^{2}=4,348\right.$, $p=0,037)$. Além disso, o CC foi significativamente mais freqüente nas mulheres com 40 anos ou mais $\left(\chi^{2}=16,875\right.$, $p=0,001$ ) (Tabela 1).

\section{SCUSSÃO}

No presente trabalho, demonstramos uma freqüência de alterações celulares e glandulares no epitélio cérvico-vaginal nos anos de 2001 a 2003 de 3,3\%. Uma ocorrência maior foi descrita em mulheres residentes no estado do Maranhão e Acre que apresentaram respectivamente $7 \%$ e $6,4 \%$ de alterações epiteliais no exame citopatológico (13-14). Essa diferença, provavelmente está relacionada a maior procura, e conseqüentemente, mais diagnósticos dessas alterações nas mulheres 
motivadas por campanhas de combate ao CC no Estado do Maranhão e Acre.

Verificamos no presente estudo uma maior ocorrência de diagnósticos de ASCUS/AGUS e infecção pelo HPV nos esfregaços cérvico-vaginais de mulheres com menos de 40 anos. Em conformidade com os nossos achados, outro estudo demonstrou uma freqüência aumentada de lesões préneoplásicas em mulheres adolescentes e adultas. Um aumento linear dessas alterações nos últimos anos, especialmente, nas adolescentes também foi descrito ${ }^{(14)}$.

Em relação às alterações neoplásicas, demonstramos uma freqüência significativa de NIC III no grupo de mulheres com menos 40 anos, já o CC foi significativamente maior nas mulheres com 40 anos ou mais. Estudo semelhante demonstrou freqüência aumentada em cinco vezes de NIC nas mulheres adultas em relação as adolescentes (15). Em concordância, outro estudo verificou em $6,4 \%$ dos esfregaços lesões precursoras para o CC em mulher na faixa etária inferior a esperada para a doença ${ }^{(16)}$.

O fato de haver, em nosso estudo, um maior número de mulheres jovens apresentando ASCUS/AGUS e infecção HPV está de acordo com dados da literatura que demonstraram que mulheres mais jovens apresentam mais alterações préneoplásicas ${ }^{(17)}$. Provavelmente, esses resultados se devem à maior exposição dessas mulheres aos fatores de riscos que levam o desenvolvimento do CC.

Sabemos que os programas de prevenção têm como público alvo mulheres jovens em idade fértil e, portanto, mais suscetíveis a desenvolver lesões cervicais pré-neoplásicas ou neoplásicas. O exame citopatológico cérvicovaginal auxilia no diagnóstico precoce de lesões, favorecendo ao tratamento antes que a neoplasia cervical maligna se desenvolva, contribuindo dessa maneira para a diminuição da morbidade e mortalidade, já que o período de evolução de uma lesão pré-neoplásica para uma forma invasiva é relativamente longo.

Consideramos que as ações do Programa de Combate ao Câncer do Ministério da Saúde apresentam um papel fundamental no sentindo de promover o diagnóstico precoce das lesões pré-neoplásicas

contribuindo conseqüentemente para a diminuição da morbi-mortalidade por CC no Brasil.

\section{CONCLUSÃO}

Concluímos que os diagnósticos das alterações no epitélio cérvico-vaginal de acordo com a faixa etária das mulheres foram semelhantes aos já descritos na literatura. A infecção pelo HPV, principal agente carcinogênico, acometeu mulheres mais jovens, sendo as neoplasias invasivas mais freqüentes a partir da quarta década de vida.

Para o sucesso da detecção precoce das lesões precursoras e da cura das mulheres com diagnóstico de CC é necessária uma grande mobilização para detectar aquelas que apresentam fatores de risco e promover o acompanhamento periódico, através do exame citopatológico. Com base nisso, reforçamos o papel essencial exercido pelo enfermeiro no sentido de orientar e estimular essas mulheres na realização do exame, de realizar o seguimento das mulheres com diagnóstico de CC e de preservar a qualidade de vida e a saúde das mulheres em sua totalidade, através de uma assistência realizada de forma 
personalizada e humanizada. Além disso, de acordo com o presente estudo, os programas preventivos devem contemplar tanto as mulheres mais jovens quanto as na fase do envelhecimento, onde se concentra a proporção de lesões mais graves.

\section{REFERÊNCI AS}

1. World Health Organization. WHO Programme on Cancer Control. Cervical Cancer Screening in Developing Countries. Geneva: WHO. 2002. p.16.

2. Zhao EF, Bao L, Li C, Song L, Li YL. Changes in epidemiology and clinical characteristics of cervical cancer over the past 50 years. Journal of First Military Medical University $2005 ; 25$ (6): 605-609.

3. Sepúlveda C, Prado R. Effective cervical cytology screening programmers in middleincome countries: The Chilean experience. Cancer Detection and Prevention 2005 Sep; 29 (5): 405-411.

4. Aguilar-Pérez JAA, Leyva-López AG, Nájera DA, Salinas A, Lazcano-Ponce, EC. Tamizaje em câncer cervical: conociemento de la utilidad y uso citologia cervical en México. Revista de Saude Publica 2003 Fev; 37 (1): 100-106.

5. Hoffman M, Cooper D, Carrara H, Rosenberg L, Kelly J, Staner I, Williamson AL, Denny L, Toit D, Shapiro S. Limited Pap screening associated with reduced risk of cervical cancer in South Africa. International Journal of Epidemiology 2003 Aug; 32 (4): 573-577.

6. Coker AL, Du XL, Fang S, Eggleston KS. Socioeconomic status and cervical cancer survival among older women: Findings from the SEER-Medicare Linked data cohorts. Gynecologic Oncology 2006 Aug; 102 (12): 278-284.

7. Ministério da Saúde. Instituto Nacional do Câncer. Estimativa 2006 da Incidência de Câncer no Brasil. [cited 2006 abr 04] Rio de Janeiro. Available from: URL: http://www.inca.gov.br/estimativa/2006.

8. Ministério da Saúde. Programa Nacional de Controle do Câncer do Colo Uterino. Diretrizes, Estratégias e Resultados Brasília (DF); [cited 2006 abr 06] 2002. Available from: URL: http://www.inca.gov.br/prevencao/programas/ viva mulher.

9. Trottier H, Franco EL. The epidemiology of genital human papillomavirus infection. Vaccine 2006 Mar; 30 (24 Suppl): 1: S1-15.
10. Souto $R$, Falhari JPB, Cruz AD. O Papilomavirus Humano: Um fator relacionado com a formação de neoplásicas. Revista Brasileira de Cancerologia 2005 Dec; 51 (2): 155-160.

11. Baseman JG, Koutsky LA. The epidemiology of human papillomavirus infections. Journal of Clinical Virology 2005 Mar; 32 (1 Supll): S16-S24.

12. Manos MM, Kinney WK, Hurley LB. Identifying women with cervical neoplasia: Using human papilomavirus DNA testing for equivocal Papanicolaou results. Journal of the American Medical Association 1999 May, 281 (17): 1605-1610.

13. Nascimento MDSB, Pereira ACS, Silva AMN, Silva LM, Viana GMC. Programa Nacional de Combate ao Câncer de Colo Uterino no Estado do Maranhão: Análise de aspectos citológicos e epidemiológicos. Acta Oncológia Brasileira 2003 Out-Dez, 23 (3):530-535.

14. Leal EAS, Leal Junior OS, Guimarães $M H$, Vitoriano MN, Nascimento TL, Costa OLN. Lesões precursoras do câncer de colo em mulheres adolescentes e adultas jovens do município de Rio Branco-Acre. Revista Brasileira de Ginecologia e Obstetrícia 2003 Mar; 25 (2): 81-86.

15. Longatto Filho A, Etlinger D, Gomes NS, Cruz SV, Cavalieri. Freqüência de esfregaços cérvico-vaginais anormais em adolescentes e adultas: revisão de 308.630 casos. Revista do Instituto Adolfo Lutz. 2003 Abr; 62(1): 31-34.

16. Ferreccio C, Prado RB, Luzoro AV, Ampuero SLI, Snijders PJF, Meijer CJLM, et al. Population-based prevalence and age distribution of human papillomavirus among women in Santiago, Chile. Cancer Epidemiology, Biomarkers \& Prevention 2004 Dec; 13 (12): 2271-2276.

17. Moscicki AB. Impact of HPV infection in adolescent populations. The Journal of Adolescent Health 2005 Dec; 37 (6 suppll):S3S9.

Artigo recebido em 07.03 .07

Aprovado para publicação em 10.12.07 